\title{
FINITE GRAPHS OF VALENCY 4 AND GIRTH 4 ADMITTING HALF-TRANSITIVE GROUP ACTIONS
}

\author{
DRAGAN MARUŠIČ and ROMAN NEDELA
}

(Received 1 May 1998; revised 1 August 2001)

Communicated by B. McKay

\begin{abstract}
Finite graphs of valency 4 and girth 4 admitting 1/2-transitive group actions, that is, vertex-and edge- but not arc-transitive group actions, are investigated. A graph is said to be 1/2-transitive if its automorphism group acts 1/2-transitively. There is a natural orientation of the edge set of a $1 / 2$-transitive graph induced and preserved by its automorphism group. It is proved that in a finite 1/2-transitive graph of valency 4 and girth 4 the set of 4-cycles decomposes the edge set in such a way that either every 4-cycle is alternating or every 4-cycle is directed relative to this orientation. In the latter case vertex stabilizers are isomorphic to $\mathbb{Z}_{2}$.
\end{abstract}

2000 Mathematics subject classification: primary 05C25, $20 \mathrm{~B} 25$.

\section{Introduction}

Throughout this paper by a graph we mean a finite, simple and undirected graph. Given a graph $X$ we let $V(X)$ and $E(X)$ be the vertex set and the edge set of $X$, respectively. Each edge $u v=\{u, v\}$ of $X$ gives rise to two $\operatorname{arcs}(u, v)$ and $(v, u)$ of $X$. Any choice of precisely one of these two arcs for all edges of $X$ results in an oriented graph whose underlying graph is $X$. (By an oriented graph we therefore mean an ordered pair $(V, A)$, where $V$ is a finite nonempty set and $A$, the set of arcs, is an antisymmetric relation on $V$.) Unless stated otherwise graphs are assumed to be connected. Furthermore, all groups are assumed to be finite. For graph-theoretic and group-theoretic terms not defined here we refer the reader to $[3,4,18]$.

The first author was supported in part by 'Ministrstvo za znanost in tehnologijo Slovenije', project no. J1-7035-0101-96.

The second author was supported in part by the Slovak grant agency, project no. 1/339/92.

(C) 2002 Australian Mathematical Society 1446-7887/2000\$A2.00+0.00 
We are going to adopt the terminology and notations of $[10,11]$ pertaining to the particular concept of 1/2-transitive group actions on graphs. A graph $X$ is said to be vertex-transitive, edge-transitive and arc-transitive, respectively, if its automorphism group Aut $X$ acts vertex-transitively, edge-transitively and arc-transitively. Further, a graph is 1-regular if its automorphism group acts regularly on its arc set. It follows from [16,7.53, page 59] that the automorphism group of a vertex- and edge-transitive but not arc-transitive graph must necessarily have two orbits on the arc set, with each orbit containing an arc corresponding to each edge; that is, in the terminology of [18, page 24], it acts 1/2-transitively on the arc set having two orbits (of equal length). We shall thus say, although in a slight discord with the above mentioned meaning [18, page 24], that a graph $X$ is $1 / 2$-transitive provided it is vertex-and edge- but not arc-transitive. More generally, by a 1/2-transitive action of a subgroup $G \leq$ Aut $X$ on $X$ we shall mean a vertex-and edge- but not arc-transitive action on $X$. In this case we shall say that the graph $X$ is $(G, 1 / 2)$-transitive.

Recently there has been an outburst of papers dealing with the structure and classification of 1/2-transitive graphs $[1,2,5,7,8,9,10,11,12,13,14,15,17,19]$. In this paper we continue the study of $1 / 2$-transitive group actions on finite graphs of valency 4 initiated in [10], where some general results were obtained, including classification results on 1/2-transitive graphs of valency 4 enjoying certain additional structural properties, and continued in [11], where the interplay of regular maps, 1-regular graphs and graphs of valency 4 admitting 1/2-transitive group actions was studied. It follows from [13] that graphs of valency 4 and girth 3 admitting a 1/2-transitive group action are in a one-to-one correspondence (via the line graph construction) with cubic graphs having a subgroup of automorphisms acting regularly on the arc set. In particular, 1/2-transitive graphs of valency 4 and girth 3 are in a one-to-one correspondence with cubic 1-regular graphs. Our aim is to prove a similar result for graphs of valency 4 and girth 4 admitting a 1/2-transitive group action.

Let $X$ be an oriented graph. A path $P$ of $X$ is called directed if every vertex of $P$ of valency 2 is the tail of one and the head of the other of its two incident arcs. Similarly, a directed cycle in $X$ is a cycle whose every vertex is the tail of one and the head of the other of its two incident arcs. An even length cycle $C$ in $X$ is a parallel cycle if it may be decomposed into two arc-disjoint directed subpaths of equal length intersecting in two vertices, one of which is the tail and the other the head of the corresponding incident arcs. An even length cycle $C$ in $X$ is an alternating cycle if every other vertex of $C$ is the tail and every other vertex of $C$ is the head of its two incident arcs.

Let $X$ be a graph of valency 4 admitting a 1/2-transitive action of some subgroup $G \leq$ Aut $X$. Let us assign an orientation, fixed from now on, to a given edge of $X$. Then, via the $1 / 2$-transitive action of $G$, this orientation extends uniquely to an orientation of the edge set of $X$, thus giving rise to an oriented graph whose every vertex has two predecessors and two successors and whose underlying undirected 
graph is $X$. Concepts peculiar to oriented graphs, such as directed, alternating and parallel cycles, may thus be extended to 1/2-transitive graphs, via the above orientation of the edge set induced by its automorphism group. We may now state one of the two main results of this paper.

THEOREM 1.1. Let $X$ be a 1/2-transitive graph of valency 4 and girth 4 . Then the set of 4-cycles of $X$ decomposes the edge set $E(X)$ and, furthermore, either

(i) every 4-cycle is alternating or

(ii) every 4-cycle is directed.

Moreover, in case (ii) the vertex stabilizer (Aut $X)_{v}, v \in V(X)$, is $\mathbb{Z}_{2}$.

In order to prove Theorem 1.1,1/2-transitive group actions on graphs of valency 4 and girth 4 are studied, resulting in their characterization in Theorem 4.1. In Section 2 certain concepts arising in the study of 1/2-transitive group actions on graphs are introduced. Next, in Section 3 some exceptional arc-transitive graphs of valency 4 and girth 4 admitting a 1/2-transitive group action are dealt with, setting the stage for the proofs of Theorem 1.1 and Theorem 4.1 in Section 4. Finally, two infinite families of 1/2-transitive graphs of valency 4 and girth 4 satisfying, respectively, conditions (i) and (ii) of Theorem 1.1 are given at the end of this paper.

\section{Preliminaries}

Let $X$ be a graph and let $G \leq$ Aut $X$ act 1/2-transitively. There will be instances in our discussion where information on the vertex stabilizer $G_{v}, v \in V(X)$, is relevant. We shall say that $X$ is $(G, 1 / 2, H)$-transitive provided $H=G_{v}$ for some $v \in V(X)$.

There are two essentially different types of $1 / 2$-transitive group actions on graphs of valency 4 . Namely, given a graph $X$ of valency 4 admitting a $1 / 2$-transitive action of a subgroup $G \leq$ Aut $X$ and a vertex $v \in V(X)$, the restriction $G_{v}^{N(v)}$ of the stabilizer $G_{v}$ to the neighbourhood $N(v)=\{u, w, x, y\}$ of $v$ is isomorphic either to $\mathbb{Z}_{2}$ or to $\mathbb{Z}_{2}^{2}$. In other words, $X$ is either $\left(G, 1 / 2, \mathbb{Z}_{2}\right)$-transitive or $(G, 1 / 2, H)$-transitive, where $H=G_{v}$ contains a copy of $\mathbb{Z}_{2}^{2}$. (Note that $H$ is a 2-group.) Let $\{x, y\}$ and $\{u, w\}$ be the two orbits of $G_{v}$ on $N(v)$. In the first case, $G_{v}^{N(v)}=\langle(x y)(u w)\rangle$ and it is easy to see that the restriction homomorphism is a monomorphism and so $\left|G_{v}\right|=2$. In the second case, $G_{v}^{N(v)}=\langle(x y),(u w)\rangle$ and in general the order $\left|G_{v}\right|$ is not bounded as may be seen by the lexicographic products $C_{t}\left[2 K_{1}\right]$, where $C_{t}$ denotes the cycle of length $t \geq 3$ (see Section 3). The $G$-height $h_{G}(X)$ of $X$ is equal to $h$, where $\left|G_{v}\right|=2^{h}$, $v \in V(X)$. Moreover, we let the height $h(X)$ of $X$ be the maximum over all $G$-heights $h_{G}(X)$, where $G \leq$ Aut $X$ acts $1 / 2$-transitively on $X$.

A $(G, 1 / 2)$-transitive graph $X$ of valency 4 , where $G \leq$ Aut $X$, gives rise to two oriented graphs-with $X$ as their underlying graph-namely, the two orbital graphs 
of the action of $G$ on $V(X)$ relative to two paired suborbits of length 2. Let $D_{G}(X)$ be one of these two graphs fixed from now on. For $u, v \in V(X)$ we shall write $u \rightarrow v$ if $(u, v)$ is an arc in $D_{G}(X)$ and shall say that $u$ is a predecessor of $v$ (and the tail of $(u, v)$ ), and that $v$ is a successor of $u$ (and the head of $(u, v)$ ). We remark that by the $G$-orientation of the edges of $X$, that is, by the orientation induced by the 1/2-transitive action of $G$, we shall always mean the corresponding orientation of the edges in $D_{G}(X)$. A path $P$ in $X$ is a $G$-directed path if it is a directed path in $D_{G}(X)$. A cycle of $X$ is a $G$-directed cycle, a $G$-parallel cycle and a $G$-alternating cycle, respectively, provided it is a directed cycle, a parallel cycle and an alternating cycle in $D_{G}(X)$. In particular, if $G=$ Aut $X$ the symbol $G$ is omitted and we refer to an orientation of the edges of $X$, and to a directed path, a directed cycle, a parallel cycle and an alternating cycle of $X$. It transpires that all $G$-alternating cycles in $X$ have the same length and form a decomposition of the edge set of $X$ [10, Proposition 2.4]; half of this length is denoted by $r_{G}(X)$ and is called the $G$-radius of $X$. Moreover, any two adjacent $G$-alternating cycles of $X$ intersect in the same number of vertices and this number, called the $G$-attachment number $a_{G}(X)$ of $X$, divides $2 r_{G}(X)[10$, Proposition 2.6]. If $X$ is $1 / 2$-transitive, we let the radius and the attachment number of $X$ be, respectively, the Aut $X$-radius and the Aut $X$-attachment number of $X$. The following simple observation on attachment numbers is made in [12].

LEMMA 2.1. Let $X$ be a $(G, 1 / 2)$-transitive graph for some subgroup $G$ of Aut $X$. If the G-attachment number $a_{G}(X)$ is at least 3 then the $G$-height $h_{G}(X)$ equals 1 .

If $a_{G}(X)=r_{G}(X)$ we say that the $(G, 1 / 2)$-transitive graph $X$ of valency 4 is tightly $G$-attached. In particular, a 1/2-transitive graph $X$ of valency 4 is tightly attached if it is tightly Aut $X$-attached. A complete classification of tightly attached 1/2-transitive graphs with odd radius and valency 4 is obtained in [10, Theorem 6.2.].

By a map we mean a cellular decomposition of an orientable closed surface. A common way of constructing maps is by embedding a graph into a surface. It is well known that a map $M$ given by an embedding of a graph $X$ into a surface can be completely described by means of its rotation system, that is, by listing, for every vertex $v$ of $M$, the cyclic permutation of the incident outgoing arcs of $v$ induced by a chosen rotation of the ambient surface. We can therefore identify $M$ with the pair $(X ; R)$, where $R$ is the above rotation system for $M$. The map $M=(X ; R)$ determines the dual map $M^{*}=\left(X^{*} ; P\right)$, where $X^{*}$ is the dual graph and $P$ is the one of two possible rotations determining the geometric dual of $M$, which is consistent with the chosen orientation of the surface. We say that $M$ is positively self-dual if $M=(X ; R)$ is isomorphic to $M^{*}=\left(X^{*}, P\right)$. The map $M$ is called regular if its automorphism group Aut $M$ acts transitively (and therefore regularly) on the set of arcs of $X$. We say that a regular map $M$ is of type $\{q, p\}$, where $q, p$ are integers, if $M$ is of valency $p$ and has face-size $q$. The relationship between regular and self-dual 
maps and 1/2-transitive group actions on graphs of valency 4 is studied in [11].

\section{Exceptional graphs admitting 1/2-transitive group actions}

In this section we describe seven infinite families of arc-transitive graphs of valency 4 and girth 4 admitting a $1 / 2$-transitive group action. These graphs arise as exceptional graphs in Theorem 4.1.

A circulant is a Cayley graph of a cyclic group. Let $n$ be a positive integer and let $S=-S$ be a symmetric subset of $\mathbb{Z}_{n}$. By $\operatorname{Circ}(n, S)$ we denote the circulant with vertex set $\left\{v_{i}: i \in \mathbb{Z}_{n}\right\}$ and edges of the form $v_{i} v_{i+s}, i \in \mathbb{Z}_{n}, s \in S$. A particular family of circulants arises in the characterization of graphs of valency 4 admitting a 1/2-transitive group action with respect to which they have only two alternating cycles.

PROPOSITION 3.1 ([10, Proposition 2.4, (ii)]). Let X be a $(G, 1 / 2)$-transitive graph of valency 4 for some subgroup $G$ of Aut $X$ and let $r=r_{G}(X)$. Then $X$ has only two $G$-alternating cycles (both spanning $V(X))$ if and only if $X \cong \operatorname{Circ}(2 r,\{1,-1, s,-s\})$ for some odd $s \in \mathbb{Z}_{2 r}^{*} \backslash\{1,-1\}$ such that $s^{2} \in\{1,-1\}$. Moreover, $X$ is arc-transitive.

Let $r, t \geq 3$ be integers. For $r$ odd and $s \in \mathbb{Z}_{r}^{*}$ satisfying $s^{t} \in\{1,-1\}$, let $X(s ; t, r)$ denote the graph with vertex set $\left\{v_{i}^{j}: i \in \mathbb{Z}_{t}, j \in \mathbb{Z}_{r}\right\}$ and edges of the form $v_{i}^{j} v_{i+1}^{j+s^{i}}$, $v_{i}^{j} v_{i+1}^{j-s^{i}}\left(i \in \mathbb{Z}_{t}, j \in \mathbb{Z}_{r}\right)$. In particular, set $X(t, r)=X(1 ; t, r)$. For $r$ and $t$ even, let $Y(t, r)$ denote the graph with vertex set $\left\{v_{i}^{j}: i \in \mathbb{Z}_{t}, j \in \mathbb{Z}_{r}\right\}$ and edges of the form $v_{i}^{j} v_{i+1}^{j}, v_{i}^{j} v_{i+1}^{j+(-1)^{i+1}}\left(i \in \mathbb{Z}_{t}, j \in \mathbb{Z}_{r}\right)$. Moreover, let $Z(t, r)$ denote the graph with vertex set $\left\{v_{i}^{j}: i \in \mathbb{Z}_{t}, j \in \mathbb{Z}_{r}\right\}$ and edges of the form $v_{i}^{j} v_{i+1}^{j}, v_{i}^{j} v_{i+1}^{j+(-1)^{i+1}}\left(i \in \mathbb{Z}_{i} \backslash\{-1\}\right.$, $\left.j \in \mathbb{Z}_{r}\right)$ and $v_{-1}^{j} v_{0}^{j+r / 2}, v_{-1}^{j} v_{0}^{j+1+r / 2}\left(j \in \mathbb{Z}_{r}\right)$. It may be seen that the permutation $\rho$ mapping according to the rule $v_{i}^{j} \rho=v_{i}^{j+1}, i \in \mathbb{Z}_{t}, j \in \mathbb{Z}_{r}$, is an automorphism of $X(s ; t, r), Y(t, r)$ as well as $Z(t, r)$. Moreover, the permutation $\gamma$ defined by the rule $v_{i}^{j} \gamma=v_{i+1}^{-j}, i \in \mathbb{Z}_{t}, j \in \mathbb{Z}_{r}$, is an automorphism of both $X(t, r)$ and $Y(t, r)$. Finally, the permutation $\delta$ defined by the rule

$$
v_{i}^{j} \delta= \begin{cases}v_{i+1}^{-j}, & i \in \mathbb{Z}_{i} \backslash\{-1\}, j \in \mathbb{Z}_{r} ; \\ v_{0}^{-j+r / 2}, & i=-1, j \in \mathbb{Z}_{r},\end{cases}
$$

is an automorphism of $Z(t, r)$. Besides, $\gamma^{-1} \rho \gamma=\rho^{-1}$ and $\delta^{-1} \rho \delta=\rho^{-1}$ and so $\langle\rho, \gamma\rangle$ in the case of graphs $X(t, r)$ and $Y(t, r)$ and $\langle\rho, \delta\rangle$ in the case of the graph $Z(t, r)$ is a metacyclic subgroup of automorphisms acting vertex-transitively.

PROPOSITION 3.2. Let $X$ be a tightly $G$-attached $(G, 1 / 2)$-transitive graph of valency 4 , where $G \leq$ Aut $X$, with $r \geq 2$ and $t \geq 3$ as its respective $G$-radius and 
the number of $G$-alternating cycles. If $X$ contains a $G$-parallel 4-cycle then $X$ is isomorphic to $C_{t}\left[2 K_{1}\right]$ if $r=2$, to $Y(t, r)$ or to $Z(t, r)$ if $r \geq 4$ is even and to $X(t, r)$ if $r$ is odd.

Proof. The fact that $X$ is tightly $G$-attached is crucial. Supposing first that $r$ is odd, we have by [10, Proposition 3.3] that $X$ is isomorphic to $X(s ; t, r)$ and it is easily seen that the existence of a $G$-parallel 4-cycle in $X$ forces $s \in\{1,-1\}$ and so $X \cong X(t, r)$.

Suppose now that $r$ is even. The case $r=2$ is proved in [10, Proposition 3.1]. Let $r \geq 4$. Let $v \in V(X)$ and let $u, w$ be the two neighbours of $v$ on a given $G$-alternating cycle of $X$ containing $v$. By [10, Proposition 3.1] there exists an automorphism $\rho$ of $X$ taking $u$ to $w$ whose orbits are the $G$-attachment sets of $X$, as well as an automorphism $\sigma$ cyclically permuting the $G$-attachment sets of $X$ in such a way that $\sigma^{-1} \rho \sigma=\rho^{s}$ for some $s \in \mathbb{Z}_{r}^{*}$. For each $i \in \mathbb{Z}_{i}$, let $V_{i}$ be the $G$-attachment set containing $v \sigma^{i}$. Further, for each $j \in \mathbb{Z}_{r}$, let $v_{0}^{j}=v \rho^{j}$ and $v_{1}^{j}=w \rho^{j}$. Next, let $v^{\prime}$ be the fourth vertex in the $G$-parallel 4-cycle containing vertices $v, u$ and $w$. Of course, $v^{\prime} \in V_{2}$ and one can easily see that $u^{\prime} \rho=w^{\prime}$, where $u^{\prime}, w^{\prime} \in V_{3}$ are the other two neighbours of $v^{\prime}$. For each $j \in \mathbb{Z}_{r}$, we set $v_{2}^{j}=v^{\prime} \rho^{j}$ and $v_{3}^{j}=w^{\prime} \rho^{j}$. Continuing this way we end up with a graph having vertex set $\left\{v_{i}^{j}: i \in \mathbb{Z}_{t}, j \in \mathbb{Z}_{r}\right\}$ and edges of the form $v_{i}^{j} v_{i+1}^{j}, v_{i}^{j} v_{i+1}^{j+(-1)^{i}}\left(i \in \mathbb{Z}_{t} \backslash\{-1\}, j \in \mathbb{Z}_{r}\right)$. As for the edges with one endvertex in $V_{t-1}$ and the other in $V_{0}$, there are precisely two possibilities, one resulting in a graph isomorphic to $Y(t, r)$ and the other in a graph isomorphic to $Z(t, r)$. Namely, since $X$ is a tightly $G$-attached $(G, 1 / 2)$-transitive graph, it follows by [10, Proposition 3.1] that there exists an involutory automorphism, say $\tau$, fixing $v=v_{0}^{0}$ and fixing each of the sets $V_{i}, i \in \mathbb{Z}_{l}$, setwise. One may easily see that $\tau$ must obey the rule $v_{i}^{j} \tau=v_{i}^{-j}$, $j \in \mathbb{Z}_{r}$, for $i$ even and $v_{i}^{j} \tau=v_{i}^{-j-1}, j \in \mathbb{Z}_{r}$, for $i$ odd. Using this automorphism we then deduce that, for each $j \in \mathbb{Z}_{r}$, the neighbours of $v_{t-1}^{j}$ in $V_{0}$ are either $v_{0}^{j}$ and $v_{0}^{j+1}$ or $v_{0}^{j+r / 2}$ and $v_{0}^{j+1+r / 2}$. In the first case we have $X \cong Y(t, r)$ and in the second case $X \cong Z(t, r)$.

Let $r \geq 3$ be an integer and let $X=C_{r} \times C_{r}$ be the Cartesian product of two cycles of length $r$. There is a unique quadrilateral embedding $M$ of $X$ into the torus. If $r$ is even then we can orient the edges of $X$ in such a way that each copy of $C_{r}$ forms an alternating cycle and every 4-cycle of $X$ bounding a quadrilateral region in $M$ is directed in the prescribed orientation. From the planar representation of $M$ one can easily see that there is an automorphism $\rho$ of $X$ rotating a specific quadrangle and preserving the above orientation. Moreover, the vertex stabilizer (Aut $X)_{v}, v \in V(X)$, contains two reflections $\alpha$ and $\beta$ fixing, respectively, the two alternating cycles meeting at $v$. It follows that $X=C_{2 s} \times C_{2 s}, r=2 s$, is $\left(G, 1 / 2, \mathbb{Z}_{2}^{2}\right)$ transitive, where $G=\langle\alpha, \beta, \rho\rangle$. We note that, letting the vertex set of $X$ be $\mathbb{Z}_{2 s} \times \mathbb{Z}_{2 s}$, the orientation of the edges of $X$ which admits the 1/2-transitive action of the group 


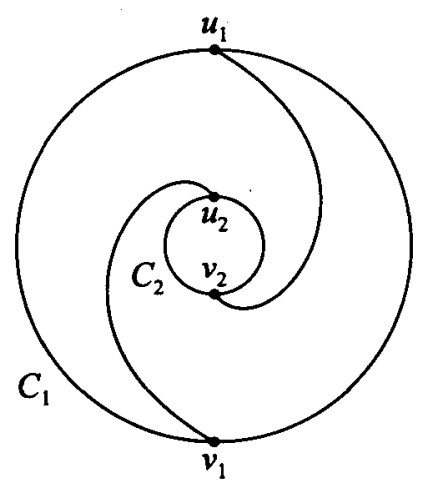

FIGURE 1.

$G$, satisfies the following rule: the vertex $(i, j)$ is a predecessor of $(i, j+1)$ and a successor of $(i+1, j)$ if $i+j$ is even, and is a successor of $(i, j+1)$ and a predecessor of $(i+1, j)$ if $i+j$ is odd.

There is another infinite family of graphs sharing similar properties with the Cartesian product of two even cycles of equal length. Let $Y=C_{2 r} \otimes C_{2 r}, r \geq 3$, denote the graph whose vertex set is the edge set of $X=C_{r} \times C_{r}$, with two vertices of $Y$ being adjacent if the corresponding edges form consecutive sides of a quadrangle in the quadrilateral embedding $M$ of $X$ into the torus. The graph $Y$ is the so called medial graph of $M$. Since the map $M$ is regular and self-dual it follows from [11, Theorem 4.1 (4)] that the graph $Y$ is $\left(G, 1 / 2, \mathbb{Z}_{2}^{2}\right)$-transitive for a particular subgroup $G$ of Aut $Y$. (This fact can also be checked directly.) Moreover, the $G$-orientation of the edges of $Y$ induced by $G$ is such that all 4-cycles of $Y$ are $G$-directed. The $G$-alternating cycles of $Y$ have length $2 r$ and two of them are either disjoint or meet in two vertices (see Figure 1 for $r=3$ ). Moreover, we let $C_{4} \otimes C_{4}$ be the lexicographic product $C_{4}\left[2 K_{1}\right]$ (which is isomorphic to the complete bipartite graph $K_{4,4}$ ). Alternatively, we may define $Y=C_{2 r} \otimes C_{2 r}, r \geq 2$, as a particular quotient graph of $X=C_{2 r} \times C_{2 r}$, obtained by identifying the vertices $(i, j)$ and $(i+r, j+r)$ of $X$, for all $i, j \in \mathbb{Z}_{2 r}$, with the adjacency as well as the orientation of the edges being inherited from that of $X$.

The next proposition follows from the above arguments.

PROPOSITION 3.3. Let $X \cong C_{2 r} \times C_{2 r}$ or $X \cong C_{2 r} \otimes C_{2 r}$ for some $r \geq 2$. Then $X$ is $a\left(G, 1 / 2, \mathbb{Z}_{2}^{2}\right)$-transitive graph for some subgroup $G \leq$ Aut $X$, and consequently $h(X) \geq 2$. Moreover, the $G$-orientation of the edge set of $X$ is such that every edge of $X$ lies on four $G$-directed 4-cycles if $X \cong C_{4} \otimes C_{4}$ and on two $G$-directed 4-cycles otherwise. 
In the next proposition we show that the converse is also true.

PROPOSITION 3.4. Let $X$ be a $(G, 1 / 2)$-transitive graph of valency 4 , for some subgroup $G \leq$ Aut $X$, such that every edge of $X$ lies on precisely two $G$-directed 4-cycles and $h_{G}(X) \geq 2$. Then either $X \cong C_{2 r} \times C_{2 r}, r \geq 2$ or $X \cong C_{2 r} \otimes C_{2 r}, r \geq 3$.

Proof. By gluing a 2-cell to every $G$-directed 4-cycle of $X$ we obtain a 4-valent 4gonal map $M$ in a surface $S$ of Euler characteristic 0 . As a consequence of transitivity of quadrangles we have that the two alternating cycles meeting at a vertex $v$ cross each other at $v$. It is well known that a simple closed curve in a surface of Euler characteristic 0 is of one of the following four types: (i) contractible-it bounds a disk, (ii) nonseparating and orientation changing, (iii) separating and noncontractible-it bounds a Möbius band, (iv) nonseparating, noncontractible and orientation preserving. We first show that every $G$-alternating cycle $C$ of $X$ is of type (iv).

Assuming that $C$ bounds a disk $D$ we get a quadrangulation of $D$ and a contradiction can be derived using Euler's formula. If $C$ is of type (ii) then the subgraph of $X$ induced by all the quadrangles incident with $C$ forms a quadrangulation of a Möbius band $B$ with the boundary of $B$ being formed by another $G$-alternating cycle whose length would be twice the length of $C$, a contradiction. Finally, if $C$ bounds a Möbius band $B$, we may assume that the number of quadrangles inside $B$ is minimal, that is, $B$ does not contain a $G$-alternating cycle separating a Möbius band. Then $B$ is a union of quadrangles incident with $C$. If $C$ meets just one side of each quadrangle of $B$, then there is a $G$-alternating cycle of type (ii) inside $B$, and a contradiction is derived as above. If $C$ meets two opposite sides of every quadrangle we take a quadrangle $Q$ in $B$ and an element $\psi \in G$ rotating $Q$. Clearly, $\psi$ maps $C$ onto some $G$-alternating cycle $C^{\prime}$ meeting $C$ in the four vertices of $Q$. Thus the two $G$-alternating cycles intersect in at least four vertices and, by Lemma 2.1, we have that $h_{G}(X)=1$, a contradiction.

Having seen that every $G$-alternating cycle of $X$ is of type (iv), we can cut and open the surface along one such cycle $C$. We get an annulus $A$ bounded by two copies $C_{1}$ and $C_{2}$ of $C$. Let us consider a $G$-alternating cycle $C^{\prime}$ meeting $C$ at a vertex $u$. Since $C^{\prime}$ is of type (iv), it follows that $S$ is a torus. By Lemma 2.1, $C^{\prime}$ meets $C$ in one or two vertices. In the first case $C^{\prime}$ is represented on $A$ by a path joining the two copies $u_{1}$ and $u_{2}$ of $u$ on $C_{1}$ and $C_{2}$. In the second case, let $v$ be the second vertex. Then $C^{\prime}$ is represented by two paths joining $u_{1}$ to $v_{2}$ and $v_{1}$ to $u_{2}$ (see Figure 2), where $u_{i}$ and $v_{i}, i=1,2$, are the respective copies of $u$ and $v$ on $C_{i}, i=1,2$. Now cutting $A$ along the paths representing $C^{\prime}$ we get the planar representations of toroidal embeddings of $C_{2 r} \times C_{2 r}, r \geq 2$, in the first case and $C_{2 r} \otimes C_{2 r}, r \geq 3$, in the second case. Moreover, note that in the second case $\{u, v\}$ is the $G$-attachment set arising from the $G$-alternating cycles $C$ and $C^{\prime}$ and so it follows that $u$ and $v$ are antipodal on both $C$ and $C^{\prime}$. 


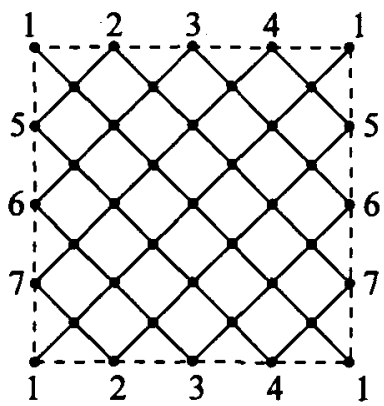

FIGURE 2.

We say that a graph of valency 4 admitting a $1 / 2$-transitive group action is exceptional if it belongs to one of the following families of (arc-transitive) graphs.

(i) the family $\mathscr{F}_{1}$ of circulants $\operatorname{Circ}(2 r,\{1,-1, s,-s\})$, where $r \geq 2$ and $s \in \mathbb{Z}_{2 r}^{*}$ satisfies $s^{2} \in\{1,-1\}$;

(ii) the family $\mathscr{F}_{2}$ of graphs $X(t, r)$, where $t \geq 3$ is an integer and $r \geq 3$ is an odd integer;

(iii) the family $\mathscr{F}_{3}$ of graphs $Y(t, r)$, where $t \geq 4$ and $r \geq 4$ are even integers;

(iv) the family $\mathscr{F}_{4}$ of graphs $Z(t, r)$, where $t \geq 4$ and $r \geq 4$ are even integers;

(v) the family $\mathscr{F}_{5}$ of lexicographic products $C_{t}\left[2 K_{1}\right]$, where $t \geq 3$ is an integer;

(vi) the family $\mathscr{F}_{6}$ of Cartesian products $C_{2 r} \times C_{2 r}, r \geq 2$;

(vii) the family $\mathscr{F}_{7}$ of graphs $C_{2 r} \otimes C_{2 r}, r \geq 3$.

Let us remark that the exceptional graphs belonging to $\mathscr{F}_{6}$ and to $\mathscr{F}_{7}$ are the underlying graphs of reflexible regular maps of type $\{4,4\}$ (see [6] for the classification of such maps).

The proof of the next proposition is left to the reader.

PROPOSITION 3.5. The following isomorphisms hold between members of different families of exceptional graphs.

(i) $C_{2 r} \times C_{2 r}, r \geq 2$, is isomorphic to $Z(2 r, 2 r)$;

(ii) $C_{2 r} \otimes C_{2 r}, r \geq 3$ is isomorphic to $Y(2 r, r)$ if $r$ is even and to $X(2 r, r)$ if $r$ is odd;

(iii) $C_{2 r}\left[2 K_{1}\right], r \geq 3$, is isomorphic to $X(4, r)$ if $r$ is odd and to $Y(4, r)$ if $r$ is even.

Let us emphasize, however, that for each of these pairs of isomorphic graphs in Proposition 3.5, the associated 1/2-transitive group actions are different.

PROPOSITION 3.6. Every exceptional graph is arc-transitive.

ProOF. In view of Proposition 3.5, it suffices to check that every graph belonging 
to one of the families $\mathscr{F}_{i}, i=1,2,3,4,5$, is arc-transitive. The arc-transitivity of lexicographic products $C_{t}\left[2 K_{1}\right] \in \mathscr{F}_{5}$ is obvious. The arc-transitivity of the circulants in $\mathscr{F}_{1}$ follows from Proposition 3.1. The arc-transitivity of the graphs $X(t, r) \in \mathscr{F}_{2}$ is deduced from [10, Corollary 3.6]. To see that the graphs $Y(t, r) \in \mathscr{F}_{3}$ and the graphs $Z(t, r) \in \mathscr{F}_{4}$ are arc-transitive, note that the permutation $\tau$ defined by the rule

$$
v_{i}^{j} \tau= \begin{cases}v_{i}^{-j}, & i \text { even, } j \in \mathbb{Z}_{r} \\ v_{i}^{-j-1}, & i \text { odd }, j \in \mathbb{Z}_{r},\end{cases}
$$

is an automorphism of $Y(t, r)$ and $Z(t, r)$. Furthermore, the permutation $\omega$ defined by the rule

$$
v_{i}^{j} \omega=v_{t-i}^{j}, \quad i \in \mathbb{Z}_{t}, j \in \mathbb{Z}_{r},
$$

is an automorphism of $Y(t, r)$ and the permutation $\vartheta$ defined by the rule

$$
v_{i}^{j} \vartheta= \begin{cases}v_{-i}^{j}, & i \neq 0, j \in \mathbb{Z}_{r} ; \\ v_{0}^{j+r / 2}, & i=0, j \in \mathbb{Z}_{r},\end{cases}
$$

is an automorphism of $Z(t, r)$. Hence the groups $\langle\rho, \gamma, \tau, \omega\rangle$ and $\langle\rho, \delta, \tau, \vartheta\rangle$, act arc-transitively on the graphs $Y(t, r)$ and $Z(t, r)$, respectively.

Let $X$ be any graph of valency 4 with the property that every edge lies on precisely two 4-cycles. A 2-path of $X$ is called straight if it does not extend to a 4-cycle. A cycle $C$ of $X$ is said to be straight if every 2-path of $C$ is straight. Using the fact that each edge of $X$ is on precisely two 4-cycles, it may be seen that an arbitrary 2-path of $X$ which is extendable to a 4-cycle is uniquely extendable to a 4-cycle. We deduce that every edge of $X$ is uniquely extendable to a straight 2-path and thus also to a straight cycle and that, moreover, at every vertex of $X$ two straight 2-paths, and therefore also two straight cycles, intersect. In particular, straight cycles form a decomposition of $E(X)$.

In view of the above comments, the proof of the next lemma is straightforward.

LEMMA 3.7. Let $X$ be a graph of valency 4 such that each of its edges lies on precisely two 4-cycles. Then the straightness of cycles of $X$ is preserved by Aut $X$. Moreover, if any two adjacent straight cycles of $X$ meet in at least three vertices, then the only automorphism of $X$ fixing two adjacent vertices is the identity.

PROPOSITION 3.8. Let $X$ be an exceptional graph. Then

(i) $h(X)=h \geq 3$ if and only if $X \cong C_{h+1}\left[2 K_{1}\right]$ and $h \geq 3$;

(ii) $h(X)=2$ if and only if $X$ is isomorphic either to $C_{3}\left[2 K_{1}\right]$ or to $C_{2 r} \times C_{2 r} \cong$ $Z(2 r, 2 r)$ for some $r \geq 2$, or to $C_{2 r} \otimes C_{2 r} \cong X(2 r, r)$ for some $r \geq 3$ odd or to $C_{2 r} \otimes C_{2 r} \cong Y(2 r, r)$ for some $r \geq 4$ even; 
(iii) and $h(X)=1$ in all other cases.

PROOF. Suppose first that $X=C_{t}\left[2 K_{1}\right]$ for some $t \geq 3$. To prove $h(X) \geq t-1$ let us take one of the two possible directed orientations of the base cycle $C_{t}$ and extend it consistently to the edges of $X$. It is easily seen that the subgroup $G$ of the orientation preserving automorphisms of $X$ acts $\left(G, 1 / 2, \mathbb{Z}_{2}^{t-1}\right)$-transitively on $X$. On the other hand, the vertex stabilizer (Aut $X)_{v}, v \in V(X)$, has order $2^{t}$. But Aut $X$ acts arc-transitively, forcing $h(X)<t$ and so $h(X)=t-1$. (Of course, $h(X)=3$ if $X=C_{4} \otimes C_{4}$ and, by Proposition 3.5, we have $h(X)=2 r-1>3$ if $X$ is either $X(4, r), r \geq 3$ odd, or $Y(4, r), r \geq 4$ even).

If $X=C_{4} \times C_{4} \cong Z(4,4)$, then one can easily see that the vertex stabilizer acts faithfully on the set of neighbours, thus forcing $h(X)=2$, in view of Proposition 3.3.

Next, suppose that $X=\operatorname{Circ}(10,\{1,-1,3,-3\})$. It is easy to see that $X \cong$ $K_{5,5}-5 K_{2}$. Assuming that $X$ is $(G, 1 / 2)$-transitive for some $G$, it follows that the $G$-orientation of the edges determines a decomposition $\mathscr{D}$ of $E(X)$ into alternating cycles (of even length). Since $X$ has 20 edges, $\mathscr{D}$ either consists of two 10-cycles or it consists of five 4-cycles. In the first case $h_{G}(X)=1$ by Lemma 2.1. In the second case we define a new graph $Y$ whose vertices are the alternating 4-cycles, two being adjacent if the corresponding 4-cycles share a vertex in common. But two alternating 4-cycles can have at most one vertex in common. Consequently, $Y \cong K_{5}$. Moreover, $Y$ admits a 1/2-transitive action of the group $G$, the latter giving rise (via the corresponding alternating cycles) to a decomposition of $E(Y)$ into even length cycles, which is clearly not possible as $Y$ has 10 edges. This contradiction shows that $h(X)=1$.

We may now assume that $X$ is neither $C_{t}\left[2 K_{1}\right], t \geq 3$ nor $X(4, r), r \geq 3$ odd, nor $Y(4, r), r \geq 4$ even, nor $\operatorname{Circ}\left(10,\{1,-1,3,-3\}\right.$ nor $C_{4} \times C_{4} \cong Z(4,4)$. We first show that $h(X) \leq 2$. Observe that every edge of $X$ lies on exactly two 4-cycles. This implies that the action of the vertex stabilizer (Aut $X)_{v}, v \in V(X)$, acts faithfully on the set of neighbours $\left\{u_{1}, u_{2}, u_{3}, u_{4}\right\}$ of $v$. Suppose that $u_{1} v u_{2}$ and $u_{3} v u_{4}$ are the two straight 2-paths at $v$. Then the sets $\left\{u_{1}, u_{2}\right\}$ and $\left\{u_{3}, u_{4}\right\}$ are blocks of imprimitivity of (Aut $X)_{v}$. Thus (Aut $\left.X\right)_{v} \leq D_{8}$, where $D_{8}$ denotes the dihedral group of order 8 . Consequently, $h(X) \leq 2$. To compute $h(X)$, we go through a case to case analysis for each of the exceptional families $\mathscr{F}_{i}, i=1,2,3,4,6,7$.

If either $X \cong C_{2 r} \times C_{2 r} \cong Z(2 r, 2 r)$ or $X \cong C_{2 r} \otimes C_{2 r}$ for some $r \geq 3$, then $h(X)=2$ in view of Proposition 3.3.

Suppose that $X=\operatorname{Circ}(2 r,\{1,-1, s,-s\})$, where $r>5$ is odd and $s \in \mathbb{Z}_{2 r}^{*}$ satisfies $s^{2}= \pm 1$. It is easily seen that each edge of $X$ lies on precisely two 4-cycles. Also, observe that $X$ has two straight cycles, namely the Hamilton cycles $v_{0} v_{1} v_{2} \cdots v_{2 r-1}$ and $v_{0} v_{s} v_{2 s} \cdots v_{2 r-s}$ and by Lemma 3.7, we have $h(X)=1$.

Suppose now that $X=X(t, r)$, where $t \neq 4$ and $r$ odd. It follows from the 
definition of $X(t, r)$ that the intersection of the two straight cycles meeting at $v_{0}^{0}$ contains the vertex $v_{0}^{m t}$ for every $m \in \mathbb{Z}$. Since $r$ is odd, the set $\left\{v_{0}^{m t}: m \in \mathbb{Z}\right\}$ contains at most two elements if and only if $t \equiv 0(\bmod r)$. But if $t>2 r$ then the above intersection contains the vertices $v_{r}^{0}$ and $v_{2 r}^{0}$. Consequently, if $t \neq r$ and $t \neq 2 r$ we have that $h(X)=1$, by Lemma 3.7. Next, since $X(2 r, r) \cong C_{2 r} \otimes C_{2 r}$ we have $h(X)=2$ for $t=2 r$. On the other hand, note that the graph $X(r, r)$ is isomorphic to $Y=C_{r} \times C_{r}$. Assume that $h_{G}(Y) \neq 1$ for some subgroup $G$ of Aut $Y$. Then there is an element of $G$ fixing a vertex $v$ of $Y$, fixing pointwise one of the two straight cycles meeting at $v$ and reflecting the other one. Since $r$ is odd this automorphism inverts an edge, contradicting the 1/2-transitive action of $G$. Thus $h(Y)=1$ and so we have $h(X)=1$ for $t=r$.

Suppose now that $X=Y(t, r)$, where $t \neq 4$ and $r \geq 4$ is even. It follows from the definition of $Y(t, r)$ that the intersection of the two straight cycles meeting at $v_{0}^{0}$ contains the vertex $v_{0}^{(m t) / 2}$ for every $m \in \mathbb{Z}$. Since $r$ is even, the set $\left\{v_{0}^{(m t) / 2}: m \in \mathbb{Z}\right\}$ equals $\left\{v_{0}^{0}\right\}$ if and only if $t \equiv 0(\bmod 2 r)$ and it contains precisely two vertices, that is, $v_{0}^{0}$ and $v_{0}^{t / 2}$, if and only if $t \equiv 0(\bmod r)$ but $t \not \equiv 0(\bmod 2 r)$. Observe that this intersection contains the vertex $v_{r}^{r / 2}$ for all $t>r$ and the vertex $v_{2 r}^{0}$ for all $t>2 r$. Moreover, if $t=r$ this intersection contains the vertices $v_{r / 2}^{r / 4}$ and $v_{r / 2}^{3 r / 4}$ if $r / 2$ is even and the vertices $v_{r / 2}^{(r+2) / 4}$ and $v_{r / 2}^{(3 r+2) / 4}$ if $r / 2$ is odd. Hence if $t \neq 2 r$, Lemma 3.7 implies that $h(X)=1$. If $t=2 r$, then $Y(2 r, r) \cong C_{2 r} \otimes C_{2 r}$ and so $h(X)=2$.

Finally, if $X=Z(t, r), t \neq r$, we can apply an argument which emulates the one used in the previous paragraph almost word by word. The details are omitted.

\section{Main results}

THEOREM 4.1. Let $X$ be $a(G, 1 / 2)$-transitive graph of valency 4 and girth 4 , where $G \leq$ Aut $X$. Then either every 4-cycle of $X$ is $G$-alternating or every 4-cycle of $X$ is $G$-directed or $X$ is one of the exceptional graphs above. Moreover, if every 4-cycle of $X$ is $G$-directed then either $h_{G}(X)=1$ or $X$ is exceptional.

ProOF. We assign to a 4-cycle $C$ in $X$ a binary sequence of length 4, on symbols 0 and 1, by the following rule: a vertex of $C$ is labeled by 1 if it is the head of one arc and the tail of the other arc in $D_{G}(X)$ and it is labeled by 0 if it is either the head of both arcs or the tail of both arcs in $D_{G}(X)$. There are then precisely four possible binary sequences (modulo a cyclic rotation): $0000,1111,1100$ and 1010, where the first, the second and the third correspond to $G$-alternating, $G$-directed and $G$-parallel 4-cycles in $X$, respectively. We shall now analyze the structure of $X$ subject to the existence of one of these types of 4-cycles.

Case 1: X contains a 4-cycle of type 1010, that is, a G-parallel 4-cycle. 
Let $r=r_{G}(X)$ be the $G$-radius of $X$ and consider two adjacent $G$-alternating cycles $C=v_{0} v_{1} \cdots v_{2 r-1} v_{0}$ and $C^{\prime}=u_{0} u_{1} \cdots u_{2 r-1} u_{0}$ meeting at the vertex $v_{0}=u_{0}$. Let $v_{1}$ and $v_{2 r-1}$ be the two successors, and $u_{1}$ and $u_{2 r-1}$ the two predecessors of $v_{0}$. By vertex-transitivity we must have that every vertex in $X$ is contained on some 4-cycle of type 1010 as the vertex having both successors on that 4-cycle. In particular, this means that the 2-path $u_{0} u_{1} u_{2}$ is extendable to a 4-cycle of type 1010. But the fourth vertex of this cycle must be either $v_{1}$ or $v_{2 r-1}$ and it has to be a successor of $u_{2}$. Hence $u_{2}=v_{2}$ or $u_{2}=v_{2 r-2}$. In both cases it is easily deduced that the two alternating cycles $C$ and $C^{\prime}$ either coincide or have precisely every other vertex in common. (See also [10]). In the first case, by Proposition 3.1, $X$ is exceptional. In the second case, $X$ is a tightly $G$-attached graph with girth 4 and so, by Proposition 3.2, isomorphic either to $X(t, r)$ or $Y(t, r)$ or $Z(t, r)$ for a suitable $t$ or to $C_{t}\left[2 K_{\mathrm{l}}\right]$, and so exceptional. (Note that the vertex stabilizer is isomorphic to $\mathbb{Z}_{2}$ in all of these cases except when $r=2$ and $X \cong C_{t}\left[2 K_{1}\right]$ when the vertex stabilizer is isomorphic to $\mathbb{Z}_{2}^{t-1}$.)

Case 2: $X$ contains a 4-cycle of type 1100.

As in Case 1, let $r=r_{G}(X)$ and consider two $G$-alternating cycles

$$
C=v_{0} v_{1} \cdots v_{2 r-1} v_{0} \quad \text { and } \quad C^{\prime}=u_{0} u_{1} \cdots u_{2 r-1} u_{0}
$$

meeting at the vertex $v_{0}=u_{0}$. Let $v_{1}$ and $v_{2 r-1}$ be the two successors, and $u_{1}$ and $u_{2 r-1}$ the two predecessors of $v_{0}$. By vertex-transitivity we must have that every vertex in $X$ is contained on some 4-cycle of type 1100 as the vertex having both successors on that 4-cycle. In particular, this means that the 2-path $u_{0} u_{1} u_{2}$ is extendable to a 4-cycle of type 1100 . It follows that either $u_{2} u_{1} u_{0} v_{1} u_{2}$ or $u_{2} u_{1} u_{0} v_{2 r-1} u_{2}$ is such a cycle. Without loss of generality we may assume that the first possibility happens. Then $v_{1}$ is a predecessor of $u_{2}$. For symmetry reasons we have that $u_{r-2} u_{r-1} v_{0} v_{r-1} u_{r-2}$ is also a 4-cycle of type 1100. Let $w$ be a predecessor of $u_{1}$. Then either $w u_{1} u_{0} v_{1} w$ or $w u_{1} u_{0} v_{2 r-1} w$ is a 4-cycle of type 1100 . If the first possibility happens we have that $w u_{1} u_{2} v_{1} w$ is a 4-cycle of type 1010 and as in Case 1 the graph $X$ is exceptional. If the latter happens then $w=v_{2 r-2}$ and so it follows that $u_{1}=v_{2 r-3}$. Thus $C$ and $C^{\prime}$ have two consecutive vertices of $C^{\prime}$ in common and we can deduce that the two vertex sets coincide, that is, $V(C)=V(X)=V\left(C^{\prime}\right)$. As in Case 1, by Proposition 3.1, the graph $X$ is exceptional. (In fact in this case one can see that $X \cong \operatorname{Circ}(10,\{1,-1,3,-3\}$.)

Case 3: $X$ contains 4-cycles of types 0000 and 1111 , that is, G-alternating and $G$-directed 4-cycles.

If two adjacent $G$-alternating cycles have two vertices in common then the graph is tightly $G$-attached and therefore exceptional. (In fact it must be isomorphic to $C_{4}\left[2 K_{1}\right]$. Assume that two adjacent $G$-alternating cycles intersect in one vertex only. We let $Y=\operatorname{Alt}(X)$ be the graph whose vertices are the $G$-alternating cycles of $X$, with two vertices joined by an edge if and only if the corresponding $G$-alternating cycles have a common vertex. It may be seen that $Y$ admits a 1/2-transitive action of the 
group $G$ and that every edge of $X$ is contained on a $G$-directed 4-cycle. Moreover, $Y$ has half the number of vertices of $X$ and so the corresponding stabilizer of the action of $G$ on $Y$ contains a copy of $\mathbb{Z}_{2}^{2}$. But then every edge of $Y$ must lie on at least two $G$-directed 4-cycles. Let $u, v$ be a pair of adjacent vertices in $Y$ with $u \rightarrow v$ in the $G$-orientation on $Y$, and let $u_{1}, u_{2}$ and $v_{1}, v_{2}$ be the two predecessors of $u$ and the two successors of $v$, respectively. Since the vertex stabilizers $G_{u}$ and $G_{v}$ contain a copy of $\mathbb{Z}_{2}^{2}$, we may easily see that the bipartite graph induced by the sets $\left\{u_{1}, u_{2}\right\}$ and $\left\{v_{1}, v_{2}\right\}$ is regular and so isomorphic either to $K_{2,2}$ or to $2 K_{1}$. In the first case, $\left\{u_{1}, u_{2}, v_{1}, v_{2}\right\}$ induces a $G$-alternating 4-cycle in $Y$, forcing $Y \cong C_{4}\left[2 K_{1}\right] \cong C_{4} \otimes C_{4}$, and consequently $X \cong C_{4} \times C_{4}$, an exceptional graph. In the second case, we see that the edge $u v$, and hence every edge of $Y$, is contained on precisely two $G$-directed 4cycles. By Proposition 3.4, we have that either $Y \cong C_{2 r} \times C_{2 r}, r \geq 2$, or $Y \cong C_{2 r} \otimes C_{2 r}$, $r \geq 3$. Now going back to $X$ we have that $X \cong C_{4 r} \otimes C_{4 r}, r \geq 2$, in the first case, and $X \cong C_{2 r} \times C_{2 r}, r \geq 3$, in the second case. Thus $X$ is again exceptional. (Observe that, with the exception of the case $X \cong C_{4} \times C_{4}$, the vertex stabilizer of the above $1 / 2$-transitive group action on $X$, which induces alternating cycles of length 4 , is isomorphic to $\mathbb{Z}_{2}$.)

Case 4: All 4-cycles in $X$ are of type 1111, that is, G-directed.

We show that either $h_{G}(X)=1$, that is, the vertex stabilizer is isomorphic to $\mathbb{Z}_{2}$, or $X$ is isomorphic to one of the graphs $C_{2 r} \times C_{2 r}, r \geq 2$, or $C_{2 r} \otimes C_{2 r}, r \geq 3$. Clearly, $r=r_{G}(X) \geq 3$. Consider two $G$-alternating cycles $C=v_{0} v_{1} \cdots v_{2 r-1} v_{0}$ and $C^{\prime}=u_{0} u_{1} \cdots u_{2 r-1} u_{0}$ meeting at $v_{0}=u_{0}$. Let $v_{1}$ and $v_{2 r-1}$ be the two successors, and $u_{1}$ and $u_{2 r-1}$ the two predecessors of $v_{0}$. There exists a vertex $x$ of $X$ such that $v_{1} \rightarrow x$ and $x \rightarrow u_{1}$, giving rise to a directed 4-cycle $v_{0} v_{1} x u_{1} v_{0}$. If there was another vertex $y$ such that $v_{1} \rightarrow y$ and $y \rightarrow u_{1}$ then $u_{1} x v_{1} y u_{1}$ would be a forbidden 4-cycle of type 1010. Similarly, $u_{1} x v_{1} y u_{1}$ would be a forbidden 4-cycle of type 1100 if $v_{1} \rightarrow y$ and $u_{1} \rightarrow y$ or if $y \rightarrow v_{1}$ and $y \rightarrow u_{1}$. Finally, if $u_{1} \rightarrow y$ and $y \rightarrow v_{1}$ then $v_{0} v_{1} w u_{1} v_{0}$ would be a forbidden 4-cycle of type 1010. We have therefore proved that $u_{1}$ and $v_{1}$ have a unique common neighbour $x$. It follows that in $X$ a directed 2-path is extendable to a unique (directed) 4-cycle. This implies that every edge of $X$ lies on either one or two directed 4-cycles. We now show that the assumption that the vertex stabilizer $G_{v_{0}}$ is not isomorphic to $\mathbb{Z}_{2}$ forces the latter possibility.

So, assume that $G_{v_{0}}$ is not isomorphic to $\mathbb{Z}_{2}$. Then there exists $\alpha \in G$ whose restriction to $\left\{v_{0}\right\} \cup N\left(v_{0}\right)$ is the permutation $\left(v_{0}\right)\left(v_{1}\right)\left(v_{2 r-1}\right)\left(u_{1} u_{2 r-1}\right)$. If $x \alpha=x$, then we must have $x \rightarrow u_{2 r-1}$ and so $x u_{1} v_{0} u_{2 r-1} x$ is a forbidden 4-cycle of type 1010. Thus $w=x \alpha \neq x$. It follows that $v_{0} v_{1} w u_{2 r-1} v_{0}$ is another directed 4-cycle containing the edge $v_{0} v_{1}$. We conclude that the graph $X$ is specified by the characteristic property that every edge is contained on precisely two 4-cycles, both of them of directed orientation. Now the proof is completed using Proposition 3.4.

As an immediate consequence of Theorem 4.1 and Proposition 3.6 we have the 
following result.

COROLLARY 4.2. Let $X$ be a 1/2-transitive graph of valency 4 and girth 4 . Then either

(i) every 4-cycle is alternating or

(ii) every 4-cycle is directed.

Moreover, in case (ii) $X$ is of height 1 .

We are now ready to prove Theorem 1.1.

PROOF OF THEOREM 1.1. Using Corollary 4.2 together with the fact that in a $1 / 2$ transitive graph of valency 4 the set of alternating cycles decomposes the edge set [10, Proposition 2.4], we may assume that 4-cycles in $X$ are all directed. Further $h(X)=1$. We now show that the set of these cycles decomposes $E(X)$. If not then Corollary 4.2 implies that every edge of $X$ lies on $\lambda \geq 2$ directed 4-cycles. If $\lambda>2$, then $X$ is isomorphic to $C_{4}\left[2 K_{1}\right]$. Thus $\lambda=2$ and applying [11, Proposition 2.2] we conclude that $X$ is the medial graph of a regular map of type $\{4,4\}$. It follows from the classification of regular maps of type $\{4,4\}$ (see [6, page 103]) that all of these maps are positively self-dual. Hence $X$ is arc-transitive by [11, Theorem 4.1], a contradiction.

The conclusion of our analysis is that 1/2-transitive graphs of valency 4 and girth 4 fall into two disjoint classes. Graphs belonging to the first class have alternating 4cycles, in other words their radius is 2 . Recently, an infinite family of such graphs has been constructed in [9] as follows. For each $n=2 k+1 \geq 17$ let $X_{n}$ denote the Cayley graph Cay $\left(A_{n},\left\{a, a-1, b, b^{-1}\right\}\right)$, where $a$ is the $n$-cycle $(0,1, \ldots, n-1)$ and $b=t a t$ is a conjugate of $a$ by $t=(02)(47)$. Then $X_{n}$ is a 1/2-transitive graph of valency 4 and radius 2 (with the automorphism group $A_{n} \times \mathbb{Z}_{2}$ ). Graphs belonging to the second class have directed 4-cycles and their height equals 1 . It follows from the results proved in [11] that every such graph is the medial graph of an irreflexible regular map of valency 4. Controlling irreflexibility of maps, however, is not at all an easy task. Also, there are examples of irreflexible regular maps that give rise to arc-transitive medial graphs. Nevertheless, there exists an infinite family of graphs belonging to the second class above. Let $s$ be any even number and let $r=1+s+s^{2}+s^{3}$. It follows from [14, Theorem 3.1] that the graph $X(s ; 4, r)$, defined in Section 3, is 1/2-transitive. Moreover, it is obvious that its girth is 4 and that all of its 4 -cycles are directed.

\section{References}

[1] B. Alspach, D. Marušič and L. Nowitz, 'Constructing graphs which are 1/2-transitive', J. Austral. Math. Soc. Ser. A 56 (1994), 391-402. 
[2] B.Alspach and M.-Y.Xu, ‘1/2-arc-transitive graphs of order 3p', J. Algebraic Combin. 3 (1994), 347-355.

[3] N. Biggs and A. T. White, Permutation groups and combinatorial structures (Cambridge University Press, Cambridge, 1979).

[4] A. Bondy and U. S. R. Murty, Graph theory with applications (American Elsevier, New York, 1976).

[5] I. Z. Bouwer, 'Vertex and edge-transitive but not 1-transitive graphs', Canad. Math. Bull. 13 (1970), 231-237.

[6] H. S. M. Coxeter and W. O. J. Moser, Generators and relations for discrete groups (Springer, New York, 1972).

[7] S. F. Du and M. Y. Xu, 'Vertex-primitive 1/2-arc-transitive graphs of smallest order', Comm. Algebra 27 (1999), 163-171.

[8] D. F. Holt, 'A graph which is edge transitive but not arc transitive', J. Graph Theory 5 (1981), 201-204.

[9] A. Malnix̌ and D. Marušič, 'Constructing 1/2-transitive graphs of valency 4 and vertex stabilizer $\mathbb{Z}_{2} \times \mathbb{Z}_{2}$, Discrete Math. 245 (2002), 203-216.

[10] D. Marušič, 'Half-transitive group actions on finite graphs of valency 4', J. Combin. Theory Ser. $B$ 73 (1998), 41-76.

[11] D. Marušic and R. Nedela, 'Maps, one-regular graphs and 1/2-transitive graphs of valency 4', European J. Combin. 19 (1998), 345-354.

[12] D. Marušic and C. E. Praeger, 'Tetravalent graphs admitting half-transitive actions; alternating cycles', J. Combin. Theory Ser. B 75 (1999), 188-205.

[13] D. Marušǐ and M.-Y. Xu, 'A 1/2-transitive graph of valency 4 with a nonsolvable group of automorphisms', J. Graph Theory 25 (1997), 133-138.

[14] M. Šajna, 'Half-transitivity of some metacirculants', Discrete Math. 185 (1998), 117-136.

[15] D. E. Taylor and M.-Y. Xu, 'Vertex-primitive 1/2-transitive graphs', J. Austral. Math. Soc. Ser. A 57 (1994), 113-124.

[16] W. T. Tutte, Connectivity in graphs (University of Toronto Press, Toronto, 1966).

[17] R. J. Wang, 'Half-transitive graphs of order a product of two distinct primes', Comm. Algebra 22 (1994), 917-927.

[18] H. Wielandt, Finite permutation groups (Academic Press, New York, 1964).

[19] M.-Y. Xu, 'Half-transitive graphs of prime cube order', J. Algebraic Combin. 1 (1992), 275-282.

IMFM, Oddelek za matematiko

Univerza v Ljubljani

Jadranska 19

1000 Ljubljana

Slovenija

e-mail: dragan.marusic@uni-lj.si
Katedra Matematiky

Univerzita Mateja Bela

97549 Banská Bystrica

Slovensko

e-mail: nedela@bb.sanet.sk 